\title{
Italie : la réforme des lycées
}

\section{Paolo Ferratini}

Traducteur : Roger-François Gauthier et Luisa Lombardi

\section{(2) OpenEdition \\ Journals}

Édition électronique

URL : http://journals.openedition.org/ries/3664

DOI : $10.4000 /$ ries.3664

ISSN : 2261-4265

\section{Éditeur}

Centre international d'études pédagogiques

\section{Édition imprimée}

Date de publication : 15 avril 2014

Pagination : 19-22

ISBN : 978-2-85420-603-6

ISSN : $1254-4590$

\section{Référence électronique}

Paolo Ferratini, «Italie : la réforme des lycées », Revue internationale d'éducation de Sèvres [En ligne], 65 | avril 2014, mis en ligne le 15 avril 2016, consulté le 08 janvier 2020. URL : http:// journals.openedition.org/ries/3664 ; DOI : 10.4000/ries.3664

Ce document a été généré automatiquement le 8 janvier 2020

(c) Tous droits réservés 


\section{Italie : la réforme des lycées}

\section{Paolo Ferratini}

Traduction : Roger-François Gauthier et Luisa Lombardi

1 L'école italienne a fait l'objet, depuis une quinzaine d'années, de nombreuses tentatives de réformes. Le système éducatif étant resté fondamentalement inchangé depuis les années 1960, tant dans sa structure que dans les programmes scolaires, on a assisté de 1996 à 2013 à une agitation presque frénétique pour "rénover ", sous cinq majorités politiques différentes, avec autant de ministres et de commissions techniques, le tout ayant abouti à mettre sur le pied de guerre les corporations et les centrales syndicales, rejointes dans la rue par les organisations d'étudiants et de lycéens.

2 Pourtant, les innovations majeures qui avaient été proposées pendant cette vague réformiste sont en fait restées lettre morte dans le texte des lois : ce fut le cas de l'autonomie des écoles et établissements, entrée dans la Constitution mais rendue impraticable en raison du manque de transfert effectif de compétences et de ressources; ce fut le cas de la réforme pour la valorisation de l'apprentissage, qui n'a jamais démarré ; ce fut le cas de la mise en place de filières d'enseignement supérieur technique court, restée au stade des bonnes intentions ou, au mieux, de mises en œuvre éphémères et locales, incapables de renouveler le système entier. Les causes principales de cette impuissance furent les fortes résistances de la bureaucratie, le manque d'une décentralisation cohérente et la difficulté à financer les réformes avec, au contraire, la tendance à consacrer lesdites réformes à la limitation des dépenses et non à l'amélioration du système.

3 Malgré ce bilan globalement mortifiant, certains changements ont néanmoins vu le jour. Même s'ils n'ont pas introduit, par eux-mêmes, des innovations véritables dans le système scolaire, ils sont néanmoins susceptibles de créer les conditions favorables pour qu'un changement plus radical intervienne dans le futur. Nous aborderons ici la réforme des lycées ${ }^{1}$, entrée en vigueur en 2010.

4 L'enseignement secondaire supérieur général n'avait pas été réformé depuis les années 1970, le ministère de l'éducation ayant alors seulement autorisé les lycées à modifier partiellement leur offre formative et à "expérimenter" de nouvelles solutions pédagogiques. Cela a permis, par exemple, à certains lycées littéraires de proposer 
l'enseignement d'une langue étrangère pendant toute la durée du cursus (cinq années) au lieu de le limiter aux deux premières années d'études, comme le prévoyait le cadre réglementaire national. Le système, au fil des ans, était devenu un patchwork illisible: l'apparition de dizaines d'expérimentations approuvées au sein des établissements avait fini par produire une sorte de jungle, au sein de laquelle presque chaque école faisait ce qu'elle voulait. D'un côté, cela témoignait d'une extraordinaire vitalité, mais de l'autre, d'une anarchie totale: horaires gonflés de façon invraisemblable (jusqu'à 38 heures par semaine!), infléchissements les plus divers infligés au curriculum des différentes filières, que souvent il n'était même plus possible de reconnaître. Pour donner une idée de l'éclatement de l'offre de formation, quand le ministère devait chaque année organiser l'examen de fin de lycée, il devait préparer plus de huit cents sujets d'épreuves différentes! Par conséquent, une réforme venant mettre de l'ordre dans cet écheveau était attendue. L'un des buts de la réforme des lycées de 2010 (en italien, ri-ordino, "remise en ordre ») a donc été de rationaliser l'offre, en créant six filières, qui correspondent à six lycées différents : littéraire, scientifique, artistique, linguistique, musical et «des sciences humaines ». La réforme a prévu également une reformulation des programmes des six filières, dont a été chargée (entre 2008 et 2010) une commission composée de dix experts : le document final contenant les nouveaux programmes a pris le nom d'«Indications nationales » (Indicazioni), puisqu'il s'agit en fait - pour la première fois dans l'histoire de l'éducation italienne - non pas d'une liste détaillée et encyclopédique de contenus d'enseignement, mais de recommandations formulées au niveau national sur les connaissances et les compétences fondamentales qui doivent être acquises par les élèves dans chaque discipline, au sein de chacune des filières. Une large autonomie serait laissée aux enseignants, relative au choix des contenus spécifiques à transmettre, en accord avec le plan d'offre formative proposé par chaque établissement.

5 Une telle révision générale des programmes et des filières apparaît fortement novatrice sur le plan pédagogique, alors qu'elle a été en fait la résultante de l'introduction d'une volonté de faire des économies financières et non, au moins en son principe, d'améliorer le système éducatif : il manqua à la réforme, au moins au début, une justification inspirée par une visée, bonne ou mauvaise, d'amélioration qualitative de l'école.

6 L'impression qu'on retira, quand le gouvernement approuva en première lecture (juin 2009) le projet de réforme, fut qu'on voulait vraiment accréditer une affirmation paradoxale, selon laquelle la réduction des ressources financières, par elle-même, "pouvait conférer efficacité et efficience au système éducatif», comme un effet collatéral purgatif. On peut être convaincu (et nous le sommes) qu'augmenter le nombre d'heures d'enseignement n'améliore pas en soi les résultats scolaires : il reste toutefois encore à démontrer que ces résultats s'améliorent automatiquement quand on réduit ce nombre d'heures.

7 Le passage de la première à la seconde et dernière lecture des Indications (février 2010) a été crucial : la redéfinition du cadre horaire autour de cinq piliers fondamentaux (italien, mathématiques, langue étrangère, sciences, histoire) et de quatre axes culturels (linguistique, mathématique, historique, et scientifico-technologique) assure l'armature d'un core curriculum commun caractérisant les deux premières années de lycée, qui marquent également, depuis 2010, la fin de la scolarisation obligatoire. Ceci a 
rendu plausible l'équivalence théorique des compétences à la sortie de la scolarité obligatoire, indépendamment des spécificités propres au curriculum de chaque filière ${ }^{2}$.

Pour comprendre toutefois quel lycée sortira de tout cela, il faut attendre de voir quel sera le destin des «Indicazioni » nationales qui se substituent aux programmes. Or l'une des grandes caractéristiques de ce document, si on le rapproche de textes semblables publiés dans le passé, saute aux yeux : sa rédaction claire, sans tableaux ni étiquettes taxonomiques. Privilégier la lisibilité d'un italien convenable au jargon techniciste de la vulgate "bureaucratico-pédagogique » n'était pas un choix neutre, mais a répondu plutôt à l'idée qu'il faut revenir à des questions simples : qu'est-il bon de chercher à enseigner? Qu'est-ce qui est fondamental que les jeunes apprennent?

Les deux autres aspects constitutifs du document sont l'organisation des disciplines et l'absence totale et revendiquée de toute suggestion didactique et méthodologique: comme indiqué dans l'exposé des motifs des Indicazioni, la structuration en disciplines repose sur la conviction que c'est aux statuts disciplinaires, qu'il faut donc sauvegarder, qu'il appartient de garantir la possibilité de construire un profil culturel cohérent et riche. Une fois tracé le territoire du savoir « que l'école a le devoir de transmettre aux nouvelles générations", poursuit le document, on laisse à l'autonomie des établissements «la liberté de tracer des parcours de qualité, sans qu'aucune recette pédagogique se trouve imposée ». Le législateur a donc fait une sorte de vœu de chasteté méthodique, ouvrant la voie au transfert vers chaque établissement de la charge de l'innovation et du défi de l'efficacité du nouveau cadre normatif.

Le choix a été fait aussi de ne pas définir de façon stricte et déductive les rapports entre connaissances et compétences : cela a l'avantage de mettre à l'abri des impasses d'une interprétation fonctionnaliste $\mathrm{du}$ processus d'apprentissage, interprétation qui considère que l'important n'est pas l'objet de l'apprentissage en lui-même, mais le développement $\mathrm{du}$ processus qui consiste à ce que des connaissances et des compétences se mettent à «fermenter " pour répondre à des situations nouvelles. Ce même choix ne met en revanche pas à l'abri d'une lecture plate de la transmission de savoirs établis et canoniques, en déplaçant en fait le balancier qui oscille entre enseignement et apprentissage du côté du premier des deux pôles.

11 On a après coup fait justement remarquer que la structure disciplinaire des Indicazioni laisse trop dans l'implicite la nécessité de donner un sens unitaire à la construction d'un savoir ouvert à la complexité, aux croisements entre les différents domaines de connaissance, comme à la pluralité des langages grâce auxquels, au fil du temps, la civilisation humaine s'est confrontée aux grands thèmes de la connaissance et du vivre ensemble.

12 En effet, la dominante héritée de l'histoire est restée l'axe autour duquel les Indicazioni reprennent le développement de chacune des branches du savoir : le fractionnement de la connaissance en disciplines, caractéristique d'un ratio studiorum vieux de trois siècles, a prévalu sur d'autres possibilités qui s'offraient, comme une présentation par champs sémantiques, par cartes mentales ou par grands thèmes ou problèmes.

13 Mais était-il possible de produire une telle vision d'ensemble, d'autant plus neuve et stimulante? Sans doute, mais elle n'eût peut-être pas été concrètement recevable par une école organisée comme l'école italienne, avec la rigidité de ses chaires disciplinaires, sa segmentation des horaires, la médiocrité de la formation moyenne de ses maîtres et l'inexistence de véritables lieux de travail collectif. 
Il est légitime de s'interroger sur le risque qu'un tel cadre aurait pu avoir comme effet de renforcer l'inertie, au lieu de promouvoir le changement. Toutefois, au-delà du fait qu'on pouvait en effet écrire un document beaucoup plus ambitieux, tout cela aurait-il effectivement suscité l'innovation, en l'absence de vrais investissements, d'un corps enseignant correctement formé à cela et d'un système d'évaluation ayant fait ses preuves? Est-il préférable, quand on manque de bateaux pour naviguer, de fixer un cap ambitieux, ou au contraire de s'en tenir à une ligne robuste consistant à redéfinir et à clarifier la route?

15 La réponse appartient à l'école et aux initiatives des acteurs. Mais le législateur doit bien comprendre que s'il n'accompagne pas le processus de façon bienveillante, avec des ressources et des solutions à la hauteur du défi, non seulement il ne se sera en rien agi de la réforme du siècle, mais même la simple perspective de maintenir l'existant en position de fonctionnement ne sera plus assurée.

\section{NOTES}

1. NDT : En Italie, l'enseignement secondaire supérieur (cinq années d'études après le collège) est organisé en trois voies : générale, technologique et professionnelle. Les «lycées » dispensent les enseignements de la voie générale, qui est déclinée, à son tour, en différentes filières.

2. NDT : on assure, dans toutes les filières de lycée, d'une part la présence des disciplines représentatives des quatre axes culturels de l'école obligatoire (qui comprend les deux premières années de lycée), d'autre part un montant horaire cohérent concernant certaines disciplines fondamentales, qui constituent un core curriculum indépendant des filières.

\section{INDEX}

Index géographique : Italie

Mots-clés : lycée, réforme de l'enseignement, enseignement secondaire deuxième cycle

Keywords : upper secondary education, educational reform, upper secondary schools

Palabras claves : escuela secundaria (segundo nivel), reforma de la educación, enseñanza secundaria (segundo nivel)

\section{AUTEURS}

\section{PAOLO FERRATINI}

Paolo Ferratinini, docteur ès lettres italiennes, a d'abord enseigné avant d'exercer les fonctions d'adjoint chargé de l'éducation au conseil municipal de Bologne. Il a participé à la plupart des 
commissions et à la rédaction de la plupart des documents législatifs importants en matière éducative sur toute la période récente. Il a conseillé en plusieurs occasions la présidence du Conseil des ministres en matière d'éducation. Il a aussi collaboré à plusieurs revues et journaux sur les questions scolaires et été professeur invité à l'Université de Bologne, ainsi qu'à l'Université La Sapienza de Rome. 